\title{
From the library
}

\section{Remembrance of things past}

Bellow was as vivid a presence in Chicago as Henry James had been in London or Beckett was in Paris. Chicagoans regularly glimpsed him lunching at Whitehall Club on the near north side or rummaging through used books at Staver's bookshop on 57th Street. Joseph Epstein, writing in the New York Times book review, remarked on the novelist's "singular" appearance: gap-toothed, white-haired, with a nose "Gogol would have approved," he radiated a quietly charismatic presence. His eyes were his most notable feature: "they are dark brown, and go deep and seem extraordinarily young. All the more young for the fact that the rest of his face looks to have taken its full fifty-five year share of the ravages of weather, work and general tribulation.” (James Atlas. Bellow, Biography. New York: Random House, 2000:403.)

\section{Protein regulation and human diseases}

Proteins are the essential fabric of which every cell is made. Regulation of proteins as they become no longer needed or fail to fold into their compact coils is a subject of some interest.

Abnormal regulation of proteins within cells can lead to human disease in at least three ways. (1) In a number of heredity anaemias, a mutant gene leads to abnormal haemoglobin production. This in turn leads to proteasomes destroying the abnormal haemoglobin and thus anaemia results. (2) In the case of cystic fibrosis, a gene mutation that ordinarily codes for a protein that moves chloride across a cell's outer membrane is abnormal. The protein is slightly misshapen and once again proteasomes break the protein down before it can reach the cell membrane. The mucous build up that occurs in this disease is directly related to the lack of normal chloride transporters. (3) In the case of several degenerative neurological diseases including Parkinson's, Huntington's, and Alzheimer's, clumps of misfolded proteins accumulate in association with the proteasomes in certain neurons. It is not clear why the neurons in these disorders fail to destroy the abnormal proteins as they do in the previous two examples. This is a focus of much of the research investigating these diseases. However, in the case of Parkinson's disease that is inherited, recent investigations suggest it results from a mutation in a gene for a specific type of protease enzyme that causes proteins to build up in brain cells and subsequently destroy them. (Scientific American 2001;January:6873.)

Can diabetes type 1 be prevented?

Type 1 diabetes is an autoimmune disorder that affects approximately four million people in Europe and North America. It occurs as the result of the patient's own immune system attacking the islet cells of the pancreas. The inciter of this inflammatory response is yet unknown, although in some cases the disease runs in families and some investigators have suggested that a viral infection may be important in the pathogenesis.

Investigators at Bristol University are now studying heat labile enterotoxin of the $E$ coli bacterium. They have demonstrated that in rats the enterotoxin prevents the immune system from destroying the insulin producing islet cells. The enterotoxin apparently binds to the receptors on the surface of macrophages, which in turn produce chemicals that inhibit the immune system. In experimental animals a nasal spray of the enterotoxin stopped the progression of type 1 diabetes. Since the early stages of type 1 diabetes can be detected in humans via antibody screening in the blood, the Bristol team hopes that screening of high risk groups before the islet cells are completely damaged might identify a population that would be amenable to therapy with the heat labile enterotoxin. (New Scientist 2000;16 December:10.)

\section{Transient ischaemic attacks: who do you worry about?}

Transient ischaemic attacks (TIA) can take many forms and present to various physicians including ophthalmologists. It is estimated that approximately 300000 TIA occur in the USA alone. A recent study suggest that in patients over 65 years of age 1 in 15 will experience a TIA at some point. Approximately $15 \%$ of patients who experience a stroke have a history of previous TIA Preventing subsequent stroke in patients with previous TIA would seem to be a reasonable strategy for the reduction in overall stroke incidence.

In a study of patients treated in the Kaiser Permanente HMO system in northern California, the short term risk of stroke and independent risk factors of developing stroke were investigated in a group of 1707 patients diagnosed with TIA in the emergency department. This study clearly documented that TIA are ominous and carry a substantial short term risk for stroke. Within the 90 day study period, $10.5 \%$ of patients with TIA developed a stroke. This is 50 times the expected in cohort of patients of comparable age. More striking was the fact that half of the strokes occurred within 2 days of the TIA.

The second portion of the study was directed at investigating whether independent risk factors could be identified that predicted which patients with TIA were likely to develop stroke. In this study five factors were independently associated with the development of stroke. They included: (1) patients' age being greater than 60 years, (2) diabetes mellitus, (3) the duration of the TIA lasted longer than 10 minutes, (4) weakness of the arms or legs occurred during the attack, (5) speech impairment was one of the symptoms of the attack It is noteworthy that although visual symptoms were present in 222 of the TIA patients, visual symptoms did not appear to be an independent factor related to the subsequent development of stroke.

Currently, available interventions for patients with TIA, including hospitalisation and urgent carotid artery ultrasound, are expensive and may not be cost effective if universally employed. However, a select group of patients with TIA identified by this study may deserve more intensive therapy than is currently being used. (FAMA 2000;284:2901-6.)
Optical illusions: a battle between the two sides of the brain?

In the past decade, Professor John Pettigrew, a neurobiologist at the University of Queensland in Brisbane, Australia, has been investigating the underlying phenomenology of visual illusions. These studies suggest that many optical illusions may work not by deceiving the visual system but rather by making visible a natural contention between the halves of the human brain. Pettigrew has suggested that it is not just groups of neurons that compete in binocular rivalry but the rivalry between the right and left hemispheres of the cerebral cortex that underlie the perception of visual illusions. His studies have involved using ice water administered to one eardrum, which induces a caloric response and an enhanced activity of the cortex on the side of the stimulus. In other patients, a highly focused one tesla magnetic field is used to disrupt the neural activity of the hemisphere to which it is applied. These studies seem to support Pettigrew's theory that the brain is faced with conflicting or ambiguous scenes from each hemisphere and that an inner hemispheric switch oscillates between the two. In a possible clinical application of this observation, Pettigrew, who has bipolar disorder, found that his brain took 10 times longer than normal to decipher the binocular rivalry test than normal. A clinical trial is being planned to test whether this problem in processing binocular rivalry may provide a simple diagnostic test for manic depression. (Scientific American 2001;284:24-6.)

\section{Treating rather than screening the} blood supply to keep it safe

Each year more than 40 million units of blood are transfused to into human patients around the world. In most parts of the developed world the blood supply is relatively safe thanks to regular screening and elimination of donors infected with dangerous viruses, particularly HIV and hepatitis B. However, bacterial contamination of the blood supply is an increased worry as well as the possibility that an unscreened virus may emerge.

At the recent American Society of Hematology meeting in San Francisco, investigators from Dartmouth Medical Center, New Hampshire, described a new approach to treating blood to prevent transmission of infectious agents. This study takes advantage of the fact that red blood cells contain no nucleus and thus do not depend on DNA or RNA. Obviously, viruses and bacteria rely on these nucleic acids to replicate and to function. Scientists at the Vitex Corporation have developed a small molecule they call inactine that penetrates the cell membrane. Once inside the cell the inactine molecules, which are positively charged, bind to the negatively charged DNA or RNA and produce a chemical reaction that leaves these molecules unable to function. Treatment with inactine reduced the viral and bacterial contamination 10000 -fold which appears to be enough to cleanse most blood supplies. Moreover, functional studies of the red blood cells indicated that the inactine had in no way interfered with the red blood cells themselves. (New Scientist 2000;16 December:12.) 